\title{
Teaching Geometry by Integrating Ethnomathematics of Bedouin Values
}

\author{
Abu Qouder Fouze, Miriam Amit \\ Ben-Gurion University of the Negev, Beer-Sheva, Israel \\ Email: abuceoud@gmail.com,amit@bgu.ac.il
}

How to cite this paper: Fouze, A. Q., \& Amit, M. (2021). Teaching Geometry by Integrating Ethnomathematics of Bedouin Values. Creative Education, 12, 402-421.

https://doi.org/10.4236/ce.2021.122029

Received: January 6, 2021

Accepted: February 20, 2021

Published: February 23, 2021

Copyright () 2021 by author(s) and Scientific Research Publishing Inc. This work is licensed under the Creative Commons Attribution-NonCommercial International License (CC BY-NC 4.0). http://creativecommons.org/licenses/by-nc/4.0/

\begin{abstract}
Numerous researches conducted in recent decades have reported on the difficulties experienced by students learning geometry. The main reason for these difficulties is the gap between the level of instruction and the students' ability to understand and learn. In other words, the students have a low level of geometrical thinking, while the teachers are attempting to instill knowledge that is at a higher level of thinking than the current level of their students. On this background, we present in this article an ethnomathematical approach combined with Van Hiele's theory of levels of thinking, with the aim of improving the instruction of geometry by making it effective, interesting, and more successful for students. For this purpose, the article will present Van Hiele's theory and the various levels of thinking, followed by the characteristics of the stages of thinking and stages of instruction. Other perspectives on learning geometry will be presented in the fifth section, followed by an attempt to explain students' difficulties in geometry through cognitive load theory and students' attitudes toward this subject, the ethnomathematical approach and its effect on the instruction of geometry will be presented, finally the effect of integrating cultural values on learning mathematics.
\end{abstract}

\section{Keywords}

Teaching Geometry, Ethnomathematics, Abu Qouder Fouze, Bedouin Cultures, Mathematics Education

\section{Introduction}

Formal geometry is perceived as the most complex field of instruction among the various mathematics subjects taught in high school. The primary quality that distinguishes geometry from other mathematical fields and makes it difficult to teach is its deductive structure. The international organization of math teachers 
also describes formal geometry as a complex field that causes difficulties in teaching and learning, since the students are usually at a stage of development in their geometrical thinking that is lower than the level required for this subject (NCTM, 2000). A broad survey that examined the pre-existing knowledge of Israeli students prior to entering junior high school found that their achievement levels in geometry, even at the most basic level, are lower than their achievements in math. The results of the (TIMSS, 2007) international survey also showed that Israeli students are ranked low in math achievement and especially geometry and solving verbal problems (TIMSS, 2007). A series of studies on learning geometrical concepts found that students in elementary school and even high school students could not distinguish between a datum, a proposition, and a property that must be proved. In other words, students have not cognitively internalized the new geometrical concepts. Furthermore, many students tend to define geometrical concepts in a naïve and simplistic manner, oblivious to the verbal definitions of concepts. For example, students define a square according to its appearance as "a quadrangular whose sides are equal to each other and whose angles are right", rather than knowing the formal definition: "A square is a parallelogram with one right angle and a pair of adjacent, equal sides" a definition that emphasizes the connection between the square and the parallelogram, in particular, and between the square and the family of parallelograms, in general.

In such studies, researchers found that students have difficulties in identifying and constructing geometrical shapes, regardless of whether they learned formal or informal geometry.

Also they reported that there are teachers who have difficulties in understanding concepts in geometry, just as much as their students. The researchers examined how simple geometry concepts are understood among three groups: $8^{\text {th }}$-grade students, $10^{\text {th }}$-grade students, and teachers in elementary school. They found similar definitions of geometrical shapes among all three groups, leading to the conclusion that certain elements in understanding and misunderstanding concepts and the development of geometrical understanding operate similarly among individuals, regardless if they are students or teachers.

Therefore, in this article we will refer to the ethnomathematical approach as an educational-instructional approach to teaching geometry that is based on integrating cultural tools and elements that express mathematical values from the daily life of the students, as a teaching aid. We will present the importance of combining Van Hiele's theory with the ethnomathematical approach in the instruction of geometry, which assists in organizing and constructing an individualized curriculum for each student according to his or her level, making learning easier and more fun, interesting, and successful for the students as evidenced by a research we conducted and that included the development and implementation of an ethnomathematical curriculum among two groups of Bedouin students in Israel. 


\section{Theoretical Background}

Geometry is perceived as one of the most complicated mathematical areas to teach (Hoffer, 1981). In the past twenty years, numerous studies have demonstrated the existence of a significant gap between teachers' ability to teach geometry and the ability of students to understand the subject matter (Gawlick, 2002; Mayberry, 1983; Senk, 1985; Usiskin, 1982). Further, difficulties in learning geometry appear among young students as early as elementary school (Hershkowitz \& Vinner, 1983; Vinner, 1976, 1977). Such difficulties are expressed in the low level of geometrical thinking among students, according to Van Hiele's $(1959,1987)$ theory and in a low level of basic skills (Hoffer, 1981).

\subsection{Van Hiele's Theory}

Van Hiele's theory of levels of geometrical thinking was developed by a pair of Dutch mathematicians in the late 1950s. The Van Hieles attempted to explain why so many students were encountering difficulties with cognitive processes involved in geometrical thinking, and in particular regarding proofs. In 1959, Van Hiele argued that there are five hierarchical levels, but in recent years math educators as well as mathematicians, including Van Hiele himself, expressed doubts regarding the existence of the fifth level, so that nowadays the accepted practice is to refer to four levels (Van Hiele, 1987).

\subsection{The Levels of Geometrical Thinking in Van Hiele's Theory}

\section{A. Level 1: Recognition}

This is the lowest level and it is primarily visual, starting with non-verbal thinking. At the level of visual thinking, shapes are judged by their appearance. We say: "I know this is a square because that's how it looks." Children may say "It's a rectangle because it looks like a box." At this level students can learn a range of shapes, be able to identify them, and distinguish between different shapes. The shape is perceived as a whole (its components are ignored), its appearance taken at face value. The reasons given by students at this level rely on the general classification of shapes according to their external shape; at this stage, students do not yet understand the properties of the shapes.

\section{B. Level 2: Analysis}

The second level is descriptive and here shapes are no longer evaluated by their looks but by certain characteristics. For example, an equilateral triangle has properties such as equal sides, equal angles, and reflective and rotational symmetry. At this stage, language plays an important role in describing shapes, and students are able to identify and analyze the properties of shapes. Yet, although students know and recognize the properties of the shapes that they see, they cannot explain each property separately, relate them to each other, or explain how one property evolves from another. In other words, students do not understand the relationships between the properties. The reasoning of students at this stage relies on informal analysis of the properties of geometrical shapes. 


\section{Level 3: Organization}

At this level, properties are organized logically; one property is derived from the other. Students use the properties that they already know. For example, in order to formulate definitions, students at this level can explain why each square is a rectangle. In other words, students at this level understand the logical order of shapes, the relationships between the shapes and their properties, and the importance of precise definitions. However, students do not yet grasp the significance of the deductive structure as a whole, so that they cannot prove the properties of geometrical shapes.

\section{Level 4: Deduction}

At this level students understand the significance of deduction and the role of fundamental concepts, definitions, axioms, theorems, and proofs (as links in the chain of deduction). Students can use assumptions in order to demonstrate proofs and they understand the meaning of "necessary and sufficient conditions". For instance, at this level students can use congruence theorems in order to prove theorems about rectangles. However, they do not yet understand the importance of precision or the formal aspect of deduction.

\section{E. Level 5: Precision}

At this level students understand the significance of precision. When dealing with various structures, they can undertake abstract deduction while comprehending the formal aspect of deduction. They can investigate the results created by switching from one system of axioms to another, and they can compare different strategies of demonstrating proof. Students can "discover" new theorems and methods of proving and can think about the problem of identifying the broadest context in which a certain theorem can be applied. For example, at this level students can understand how the Euclidian theorem is related to the existence of rectangles and that in other, non-Euclidian geometries, the axioms are different and therefore rectangles do not exist.

As mentioned, according to the Van Hiele theory, the development of geometrical comprehension can be hierarchically structured, whereby a partial mastering of each level is a necessary but insufficient condition for understanding the next level. In fact, the lack of mastery of previous levels will make it impossible for students to function at higher levels. Notably, students are able to function differentially in various levels regarding different concepts, due to differential levels of understanding they have developed regarding specific concepts. The Van Hiele theory is based on the assumption that the progression from one level to another is contingent upon the level of instruction more than on age of biological maturation. Different types of instruction and development of basic skills can variably affect students' progress or lack thereof.

\subsection{The Properties of Van Hiele's Levels of Thinking (Van Hiele, 1987)}

Van Hiele outlined five properties of levels of thinking in geometry as follows:

1) Fixed sequence: Students advance from one level to another in sequence. 
In other words, in order to advance to a higher level, students must master the lower level first.

2) Adjacency: Contrary to other learning theories, and especially Piaget's, Van Hiele's theory is based on the assumption that advancement from one level to another depends more on instruction than on age or biological maturation. Therefore, different types of instruction can affect advancement in different ways.

3) Distinction: A concept that was internalized or assimilated at one level becomes the subject of instruction in the next level. For example, in the first level the general pattern of geometrical shapes is taught, while in the second level shapes are defined by their properties.

4) Separation: Each level is distinguished by its characteristic linguistic symbols. For example, "square" is the simplest linguistic symbol given to a complex quadrangular. However, a rectangle, parallelogram, or rhombus also fit this definition. Only a student at the third level (organization) can understand this, while students at lower levels find it difficult.

5) Attainment: A student at one level will have difficulties understanding the context and vocabulary of a higher level. For example, teaching geometry as a deductive system (level 4) to students that are not even at level 3 will not bring them to level 4. At best, they will remember the propositions and their proofs, but they will not understand them and certainly will be unable to prove problems on their own.

\subsection{Stages of Instruction in the Van Hiele Theory}

Van Hiele believed that in order to maintain a successful and effective instruction-learning process, instruction should be in phases according to the level of thinking of the students, from lower to higher levels, with each stage including specific instructional activities (Van Hiele, 1987).

The first stage is called information or inquiry. In this process, teacher and student initiate a discussion or activity regarding the subject being taught. They raise questions and the teacher must present the vocabulary that characterizes this level. The purpose of this phase is for the teacher and student to recognize the initial knowledge the student has, while the student must also be introduced to the subjects to be learned and examine how his current knowledge can contribute to learning them. The second stage is directed orientation. Here the student studies a certain subject through activities presented by the teacher that should lead the student to understand the characteristics of that level of geometrical thinking. The third stage is explanation. On the basis of the initial activities conducted by the students, they may change their point of view regarding the geometrical shapes they are studying. The fourth stage is free orientation. Students cope individually or in small groups with increasingly complex tasks, constructed hierarchically. The fifth stage is integration. Here the students survey and summarize what they learned, with the aim of creating an overall picture of the subject that was taught. The teacher can assist her students in forming this 
synthesis by presenting a general and comprehensive review.

Van Hiele suggested that throughout these stages, teachers must plan tasks, direct the children's attention to the geometrical properties of shapes, present terminology, conduct discussions with the children while using these terms, and encourage explanations and problem-solving methods that utilize the descriptive language of children regarding shapes. Going through these five stages with materials that are appropriate for each stage enables children to construct a rich background in visual and descriptive thinking that includes various shapes and their properties. The stages of development of geometrical thinking are successive and children must go through all of them. An important point emphasized by the Van Hieles is that the stages are not age-dependent and that instruction and experiencing geometry is more important than age. In other words, it is important to match instruction to the level of thinking of the students in order to develop their geometrical thinking.

Most common teaching methods make use of these stages. Since Van Hiele presented his theory in 1959, and also after the change the theory underwent in 1986, several studies have been conducted around the world for the purpose of examining the validity of his model. For example, Usiskin (1982) found that the level of thinking of students can be determined, but it is difficult to reliably categorize students who are transitioning from level 1 to level 2. Usiskin also found that most high school students and pre-service teachers have not mastered the Van Hiele stages beyond stage 3, even after studying geometry. Mayberry (1983) found that most high school students do not reach stage 4 and that students can be at different levels for different subjects in the curriculum. Fuys and Gedds (1984) conducted a study among sixteen 6th graders and sixteen 9th graders and found that among the 6th graders, three were at level 1, five were transitioning from level 1 to level 2, and eight had transitioned from level 2 to level 3. Among the 9th graders, two were at level 1 , seven were at level $1-2$ but were categorized as level 2, and seven began at level 2 and were categorized as level 2 - 3 despite occasionally regressing to level 1 thinking.

Burger \& Shaughnessy (1986) found that high school students have incomplete notions of basic geometric shapes and their properties, which explains part of the frustration of students and teachers of geometry: The students do not have deep enough roots in order to understand Euclidean geometry. The students and teachers are operating on different Van Hiele levels of thinking, and this is a major source of misunderstanding between teachers and students. The researchers also found that students were liable to regress from the original stage to lower levels of thinking a year later. In fact, the responses of the students before and after beginning to learn geometry were similar, except that the latter made a better use of the vocabulary of geometry. The research also found that the Van Hiele levels of thinking are indeed hierarchical, with students moving sequentially from one stage to another, without skipping any of them. It also appears that the levels are not global or discrete; in other words, a student can be at different levels of thinking vis à vis different geometrical content and can even 
move from one stage to another within the same task.

Gutierrez et al. (1991) also found that the Van Hiele levels of thinking are not discrete, based on student responses that showed partial mastery of two adjacent levels at the same time. They also found that there are five levels of mastery in each level of thinking: no mastery, a low level of mastery, a medium level, a high level, and complete mastery. Initially, students are unaware of the existence of methods of thinking that characterize the new level and therefore do not have any mastery of this level of thinking. Once they become aware of the methods of thinking that characterize a given level, they attempt to use them. They make several attempts to work at this level and solve problems. Due to their inexperience their successes are few, and consequently they regress to lower levels of explanation. This is a low level of mastery. As students become more experienced, they advance to a medium level of mastery. In the absence of complete mastery of a new level, students move back and forth between the previous, lower level and the new one, until they master the method of thinking of the new level and use it without difficulty. Most researchers believe that correct and effective instruction can contribute to the development of higher levels of thinking and the promotion of geometrical understanding. Accordingly, any method of instruction that can help advance learning processes and reduce the difficulties involved in learning should be developed and encouraged.

\section{Another Perspective on Learning Geometry}

Hoffer (1981) articulated an important point of view in his essay "Geometry is more than a proof", according to which many students perceive the study of geometry as the learning of an incomprehensible and dull set of proofs. This is due to the fact that most of the time is dedicated to learning proofs as opposed to developing the basic skills required for learning this subject. Hoffer details five important skills that must be nurtured in the geometry curriculum: visualization skills, verbal skills, drawing skills, logical skills, and applied skills.

A. Visualization skills: Geometry is a subject that requires the visualization of abstract concepts. Therefore, students whose ability to visualize is underdeveloped may encounter difficulties in learning this subject.

B. Verbal ability: Language has a significant role in learning in general and in geometry as well, which requires students to formulate assumptions, axioms, definitions, description of shapes and the relations between them and so on. Language is used primarily in the service of concise, unified formulations in order to achieve the utmost precision. Students who are deficient in reading comprehension or verbal expression may have difficulties in learning geometry.

C. Drawing skills: In geometry, more than in other subjects, students need to be able to draw in order to understand the properties of shapes, the relationships among them, and proofs.

D. Logical skills: Geometry is a mathematical realm that demands the ability to think and to reason logically. For example, geometry teaches how to construct an argument hierarchically, to bolster arguments through offering reasons, un- 
derstand the differences between valid and non-valid reasons, and to distinguish between causes and conclusions. An inability to reason logically can make it difficult to solve problems in geometry.

E. Applied skills: Geometry is a theoretical model of the physical world around us. There is a reciprocal relationship between understanding the environment and understanding geometry. One can apply what is learned in geometry to the surrounding world, which in turn can be used in order to understand geometry. An inability to apply can be an impediment to learning geometry.

Hoffer combined these skills with Van Hiele's levels of learning and suggested that instruction must support student learning through both levels and skills.

\section{Student Difficulties in Understanding Geometry According to Cognitive Load Theory}

According to cognitive load theory, learning is more effective when it occurs under conditions that correspond to the structure and limits of human cognition, while referencing the load placed on working memory when engaged in learning, thinking, problem solving, or visualization (Sweller, 1988; Sweller, van Merrienboer, \& Paas, 1998).

Chandler \& Sweller (1991) distinguished between three types of cognitive load:

1) internal cognitive load, which is built into every pattern of learning and cannot be changed through instruction; 2) the cognitive load of the learner, which is manifested in the mental effort dedicated to processing, constructing, organizing, and automating information into patterns of thought; and 3) external cognitive load, which is determined by the way in which information is presented to the learner and therefore can be controlled by proper shaping of the instruction process. For instance, the manner in which information is presented can impede learning due to the external cognitive load involved, which may be too much or simply unnecessary. The visual difficulty involved in presenting a three-dimensional geometrical situation using a three-dimensional drawing places on some students an unnecessary external cognitive load, which may impede thinking and learning (Chandler \& Sweller, 1991).

In order to achieve an optimal level of learning, it is important to minimize and limit the external cognitive load caused by the presentation of information and strive instead to increase the cognitive load involved in organizing information into mental structures (Pollock, Chandler, \& Sweller, 2002).

\section{Student Attitudes toward Geometry}

An attitude is defined as a positive or negative disposition toward an object, concept or situation and also refers to the readiness to react consistently to related objects, concepts, or situations. Attitudes develop through numerous learning experiences. In researches conducted by Bloom (1984) 25 percent of the variance in academic achievements was explained by emotional characteristics of 
the students prior to learning processes. Thus, it behooves us to pay attention to the attitudes of students toward geometry. Following Bloom, we believe that individuals tend to like activities that they believe they can succeed in the future, as they were successful in the past. In other words, if a student believes that he succeeded in a prior task, it is likely that he will approach the next task with positive emotion, and vice versa.

Hoffer (1981) asked first-year undergraduates what were their least-liked mathematical subjects in high school and which were their favorite subjects. While the favorite subjects were varied, there was a consensus that geometry was the least liked subject, out of all mathematical subjects. The explanations provided by the students in this study included statements such as "I didn't understand it", "We proved axioms the whole year", "We had to memorize proofs all the time", and so on. Another study by Robert (1982) examined the correlation between achievement in geometry and attitudes toward the subject and found that students with low achievements disliked the subject and high-achieving students liked it.

Therefore, a basic fact and condition for a successful and effective learning process in geometry is to match the instruction materials and methods to the students' level of thinking and comprehension (Crowley, 1987; Thomas, 2000).

Sullivan \& Glanz (2004) believe that instruction is a complex activity related to students' motivation and cognitive and learning processes and also related to the planning of instruction by teachers and the way they shape norms in class. Sullivan emphasizes that teachers apply guiding principles of instruction when they plan lesson activities. Several studies have attempted to understand these guidelines and their pedagogical implications among teachers of geometry. For example, Keith (2000) conducted a study among student-teachers of geometry in order to examine their knowledge of content and pedagogy in several subjects in trigonometry and plane geometry. They found that the students lacked sufficient knowledge in both areas for teaching geometry.

In another study, Swafford, Jones, \& Thornton (1997) examined the effects of interventions for student-teachers intended to strengthen their knowledge of geometry and familiarity with studies concerning the cognition of geometry students. They reported significant gains among the students in content knowledge and changes in the subject matter, means of instruction, and characteristics presented by the teachers. The students themselves related these changes to the knowledge they acquired.

\section{How Can Geometry Be Taught and Learned?}

In 2000 the National Council of Teachers of Mathematics (NCTM, 2000) published a list of geometry standards for K-12. These standards describe the achievements in geometry that are expected from students of different ages and abilities and therefore they provide guidance for teachers regarding the appropriate subject matter for each topic and age range. The standards are divided in- 
to four main realms of geometrical thinking:

1) the properties of shapes, Students must be able to analyze characteristics and properties of 2- and 3-dimensional geometrical shapes and to develop mathematical reasons regarding geometrical relations.

2) location-space relationships, Students must be able to specify locations and describe spatial relations while using coordinate geometry and other representational systems.

3) transformations and symmetry, Students must be able to apply transformations and use symmetry to analyze mathematical situations.

4) visualization, Students must be able to use visualization, spatial reasoning, and geometric modeling to solve problems.

Therefore according to these standards we can summarize and say: geometry instruction must seek and identify thinking mistakes that children make in identifying shapes according to "standard shapes" or prototypes. To avoid such mistakes, students must be exposed to a wide range of shapes of different sizes, materials, angles and orientations. It is recommended to compare standard shapes to non-standard shapes in order to focus the attention of students on the essential elements of each shape. Also, it is recommended to conduct activities that facilitate student play with shapes, so that they can change them as they wish.

\section{The Ethnomathematical Approach and Its Effect on Geometry Instruction}

One of the leading and most successful approaches in the field of mathematics education is the ethnomathematical approach, in which instruction is based on the integration of cultural-educational elements that express mathematical values from the students' daily life. Numerous researches have been conducted around the world in support of the ethnomathematical approach in order to develop effective and engaging instruction and learning strategies and in order to prove their positive effect on successful student learning. The Brazilian researcher and educator, D'Ambrosio, is one of the most renowned researchers supporting the ethnomathematical approach, and he has made significant contributions to its development and dissemination throughout the world. D'Ambrosio developed and proposed a new curriculum in 1984 called "The socio-cultural basis for mathematics education" (D'Ambrosio, 1984). This proposal emphasized the importance of integrating cultural aspects of the instruction of mathematics within the learned subjects. This practice helps students absorb the materials and understand them better. He suggested recognizing three aspects: reading and writing, mathocracy and technocracy.

Through ethnomathematics which is linked to human life, learners can be more active in solving problems because they are related to their lives (D'Ambrosio, 1987).

According to D'Ambrosio (2002) educators are responsible for the learning process and therefore they must develop informal curricula that refer to the real- 
ity in which the student lives, while integrating traditional values in their cultural-educational context in the mathematical instruction and learning process.

According to Gilmer (1990) teaching math without cultural context on the pretext that it is perfect, abstract, and universal is the reason for students' declining achievements and their failure. To remedy this situation, we must find ways to help students learn about their cultural mathematics. When students are exposed to different mathematical cultural values and reflect upon them together they discover that they know more than they thought they knew when they were judging themselves by the formal, traditional mathematics. Furthermore, in this way they develop a desire to learn and their self-confidence grows. Also ethnomathematics helps them solve more complex problems (Powell \& Frankenstein, 1997). Implementing situations from the local culture in the classroom is one way in which to assist students in seeing the relevancy of math to their culture, and subsequently use this link to assist in teaching math. One project that does this is a project called "Increasing the Participation of Native Americans in Higher Mathematics", conducted in Oklahoma (Aichele \& Downing, 1985).

Many empirical studies point to the improved achievements of students from diverse ethnic and cultural backgrounds in mathematics tests after taking part in an ethnomathematics program.

In addition several researchers developed and implemented a theoretical structure to analyze student's lack of desire to participate in a cultural course for teachers (Verner, Massarwe, \& Bshouty, 2013). Participants were pre-service and in-service teachers, Arabs and Jews, religious and secular, who studied geometry through inquiry into geometric ornaments drawn from diverse cultures, and acquired knowledge and skills in multicultural education. The methodology of engagement structures recently proposed by Goldin et al. (2011) was used to analyze the emotional behaviors in the course. The research findings showed that engagement structures were a powerful tool for examining the student's lack of motivation to study math. The constructivist ethnomathematical approach highlighted the structures that matched our instructional goals and diminished those related to students' feelings of dissatisfaction and inequity. The researchers suggested a new engagement structure "Acknowledge my culture" that nurtures math education. Findings also showed that the participants perceived this type of learning as a meaningful experience that, contrary to other learning methods, enhances their positive feelings toward other students and teachers and contributed to a lively discourse among them and raised their level of motivation. Studies show that knowledge based on faith and affiliation to a group leads to cultural coexistence and inner peace (Amit, Fried, \& Abu-Naja, 2007), which in turn improve the students' self-conception and achievements.

\section{The Effect of Integrating Cultural Values on Learning Mathematics}

We believe that, educators are responsible for the learning process, which includes the development of curricula and learning strategies based on the integra- 
tion of cultural elements and values, and particularly ethnomathematical units of measurement, geometric shapes from the Bedouin woman traditionally embroidered, in math instruction. The use of units measurement and geometric shapes from the students' culture and of cultural values and previous knowledge in this endeavor may contribute greatly to the students' learning process, help them better understand the study material, raise their motivation and, ultimately, improve their achievements in math. This process may also contribute to an increase in students' sense of belonging to their immediate environment and their cultural values and traditions.

The effectiveness of math education and its effect on student achievements is high on the agenda of numerous countries (Keitel, Damero, Bishop, \& Gerdes, 1989), which is one reason why numerous researchers have developed and implemented ethnomathematics curricula. Studies that have been conducted on such curricula testify to its effectiveness in various aspects of math education.

For instance, Lipka, Wong, \& Ihrke (2013) presented findings from an educational project conducted among American Indians and American-Alaskans for the purpose of examining the effect of the local culture on math instruction for these students. The researchers worked with the elders of the Yup'ik tribe in order to include daily cultural activities in the school curriculum. Accordingly, the students initially learned in theory the traditional methods of math education and then implemented them in practice.

Similar results were found in our research among a Bedouin population in Israel. This study included the development and implementation of an ethnomathematics curriculum based on integrating Bedouin cultural values and elements for an especially constructed learning unit on the subject of units of measurement. Four 7th grade classes' two classes in the experiment and two as a control were the research population. Findings showed a clear improvement for the experimental group in various outcomes such as motivation and self-conception, which were at higher levels after the implementation of the ethnomathematics program compared to before. For the control group, these values did not change between the two measurements and in fact slightly dropped. The study also affected the student's positions toward their culture and the adults in their society, making these positions more positive (Amit \& Abu Qouder, 2017).

\subsection{Examples of Bedouin Units of Measurement}

\subsubsection{Traditional Units of Length (Table 1)}

1) Concept: مقرط العصا Read: M'krat ala'sa-Literal meaning: stick throwing distance. This term is one of the most common the Bedouins, especially the older generation. To understand this concept, it is important to clarify the most Bedouins make their living by herding sheep, goats, camels or other animals. The man in charge of the herd would generally hold a stick $(80-150 \mathrm{~cm})$ with which to direct the flock. The term covers a range of three to four kilometers in the directions indicated by the stick. In fact, it is a vector with a magnitude and a direction. 
2) Concept: شوط Read: Shoot-Literal meaning: the distance a horse rider can cover at a run in one burst without stopping. This is one of the more common measures today, and was designed for measuring particularly long distances. When we asked how far it was. One interviewee told us that it was the distance between two (ancient) towns-Lod and Ramle-or approximately $18 \mathrm{~km}$.

3) Concept: قامه Read: Kama-Literal Meaning: the height of an average person. Approximately $170 \mathrm{~cm}$. This unit of measurement was used to measure depth, camels and human.

\subsubsection{Traditional Units of Weight (Table 2)}

1) Concept: قربه Read: Kerbh-Literal meaning: vessel for carrying water or milk. The kerbh is a vessel made of goatskin for keeping milk in the tent or cooling water. This unit of measurement was used mainly for the sale of milk or its products. One kerbh is worth $30 \mathrm{~kg}$.

2) Concept: كوز Read: Kooz-Literal meaning: a Bedouin drinking vessel. This is unit do weight that has gradually faded away, especially as the Bedouin stopped using the Kooz. But it is not yet entirely gone. There are people, like my father, who still believe that the Kooz keeps water better than a refrigerator-keeping it cool but not too cold. As unit of weight, it was meant for sale of herbal medicines. Every Kooz is worth $1 \mathrm{~kg}$.

Table 1. In this exercise, students will be asked to measure the lengths of the objects in the table using three cultural tools: the Deraa', the Baa', and the Kama. Through this exercise, the students become familiar with the cultural units of length and how and when to use them.

\begin{tabular}{|c|c|c|c|c|}
\hline A number & What we measure & Deraa & Baa & Kama \\
\hline 1 & The length of the classroom door & & & \\
\hline 2 & the length of your desk in the classroom & & & \\
\hline 3 & The length of the classroom window & & & \\
\hline 4 & The distance from the classroom to the library & & & \\
\hline 5 & The distance from the classroom to the school gate & & & \\
\hline
\end{tabular}

Table 2. In this exercise, students are required to weigh the objects in the table using the Waqia, the Ratl, and the metric system (kg). The aim of this exercise is to familiarize the students with the cultural units of weight and how and when they are used. In addition, this exercise enables the students to compare the traditional, cultural tool and recognize the differences between them.

\begin{tabular}{|c|c|c|c|c|}
\hline A number & What we measure & Waqia & Ratl & $(\mathrm{kg})$ \\
\hline 1 & weight of one watermelon & & & \\
\hline 2 & The weight of your bag & & & \\
\hline 3 & The weight of your computer & & & \\
\hline 4 & The Weigh of your math book & & & \\
\hline 5 & The Weigh of ten tomatoes & & & \\
\hline
\end{tabular}


3) Concept: وقيه Read: Wakeh-Literal meaning: none. This is the most basic Bedouins unit for weight, and it is still used in many tribes today, measured with a deep plate. Some claim that there are four Wakeh in a kilogram, so that it is worth 250 grams.

4) Concept: كف Read: K'af-Literal meaning: Palm A unit of weight that measures the amount of flour that fits in the palm of the hand. This unit is not accurate, but it gives some indication of the amount of material. The K'af translates into approximately 30 grams. Two examples of exercises on the subject of units of length and weight in Bedouin ethnomathematics, from the research we conducted in 2017 (Amit \& Abu Qouder, 2017).

Example 1: Example from the subject of units of length

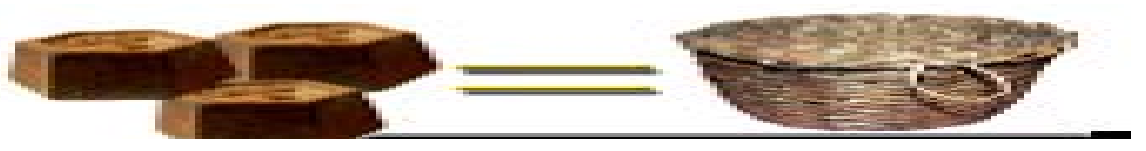

\section{Example 2: Example from the subject of units of weight}

\subsection{Examples of Geometric Shapes in Bedouin Embroidery}

The embroidery traditionally practiced by Bedouin women included numerous shapes and objects, such as flowers, plants, geometric shapes, numbers, round letters, line types, and animals all of which were hand-embroidered very precisely, using various methods and colors, several types of cloths, needles (Figures 1-6).

Examples How to integrate Bedouin embroidery in geometry instruction (Figure 7 and Figure 8)

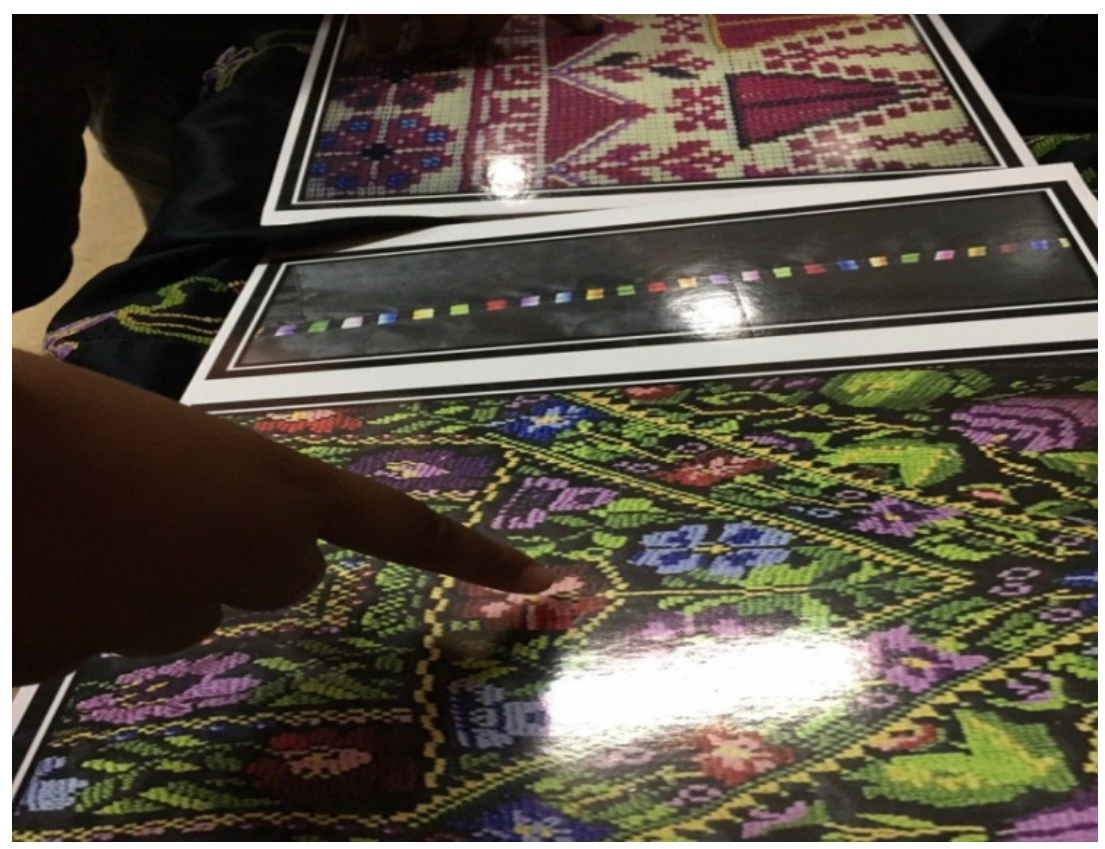

Figure 1. Example of integrating Bedouin embroidery in geometry instruction. 


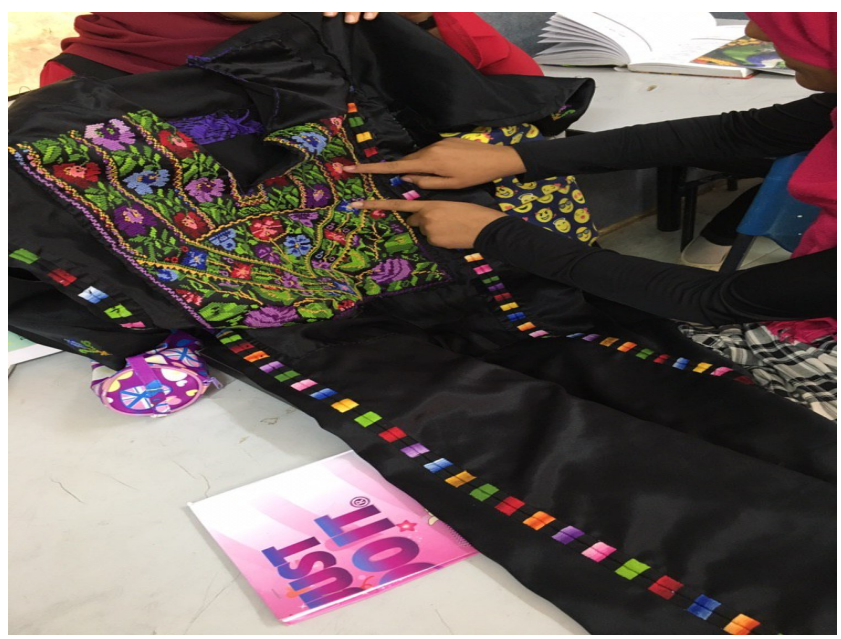

Figure 2. Example of integrating Bedouin embroidery in geometry instruction.

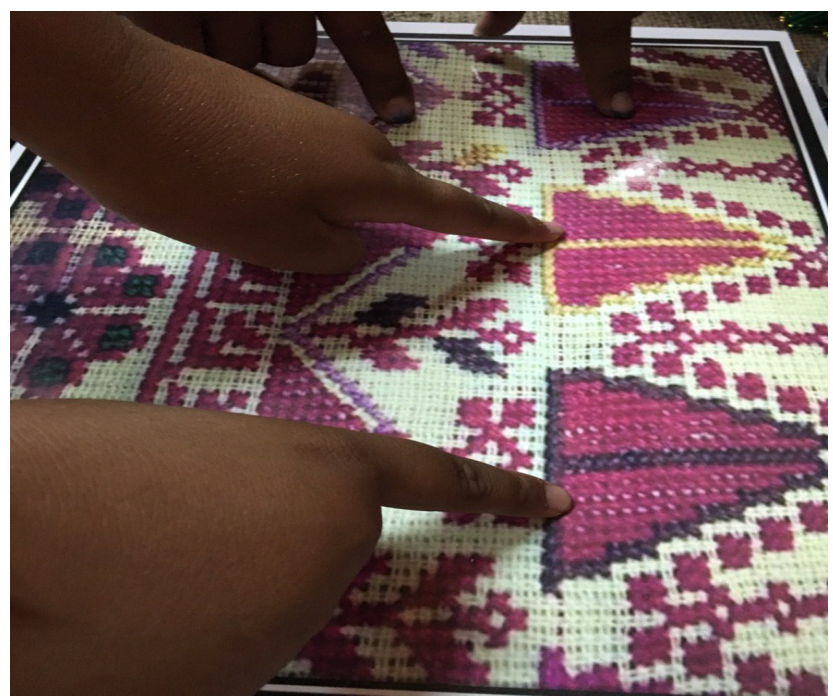

Figure 3. Example of integrating Bedouin embroidery in geometry instruction.

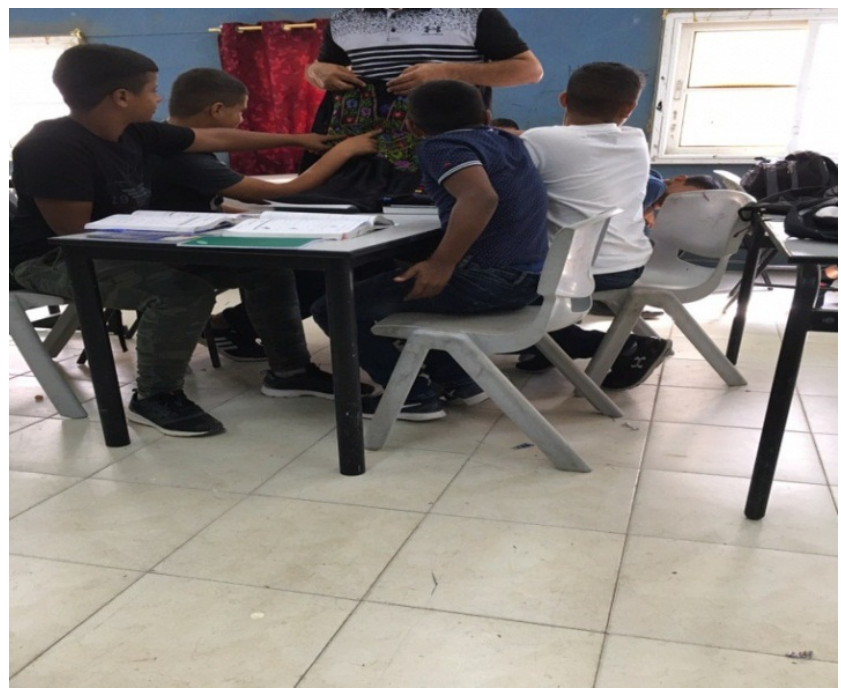

Figure 4. Example of integrating Bedouin embroidery in geometry instruction. 


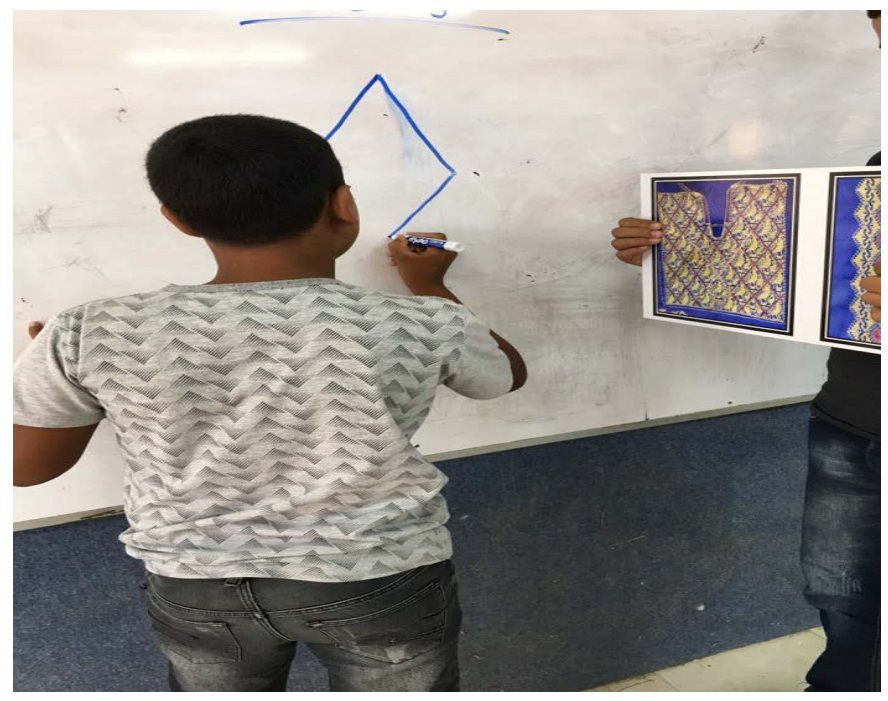

Figure 5. Example of integrating Bedouin embroidery in geometry instruction.

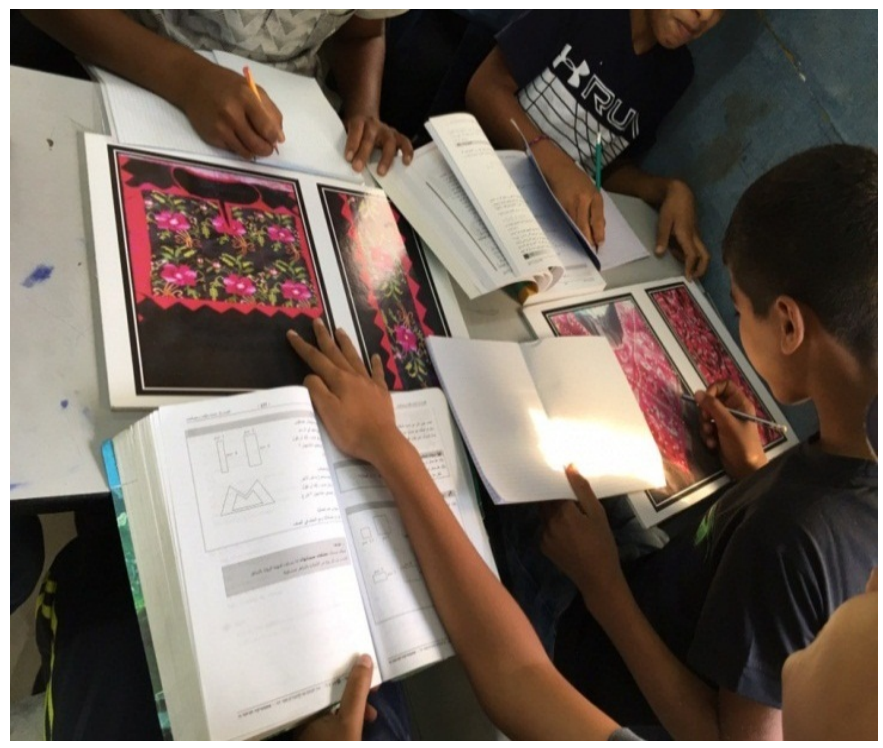

Figure 6. Example of integrating Bedouin embroidery in geometry instruction.

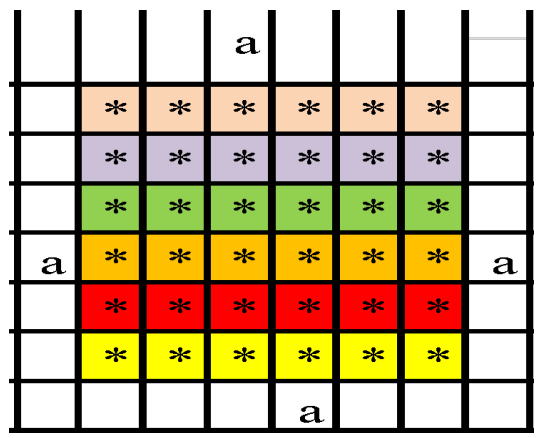

Figure 7. Illustration of a square created using the cultural method. 1) The students will be asked to draw a $6 \times 6 \mathrm{~cm}$. square on a checkered piece of paper, using a ruler. 2) The students will be asked to draw the same square on the same checkered paper, but this time using the cultural method. 


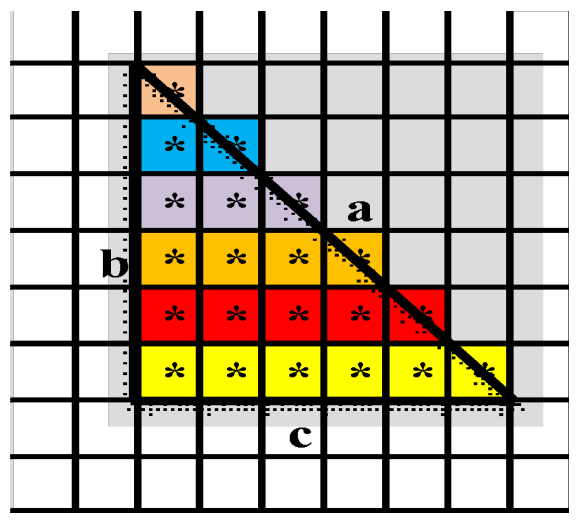

Figure 8. Illustration of a triangle created using the cultural method, according to the data in the question. For example, in one of the exercises, the students will receive a checkered paper and be asked to do the following: 1) Form a triangle on a checkered worksheet using the method of Bedouin embroidery, in a descending series of sizes $(6+5+4+3+$ $2+1$ ) as follows: first use the vertical row of six squares marked by a star, then the vertical row of five squares, followed by the vertical rows of four, three, two, and one. 2) What type of triangle was formed in exercise 1? 3) Measure the sides of the triangle in units of centimeters, using a ruler. 4) Draw a triangle using a ruler on checkered paper, according to the size of the triangle created using the method of Bedouin embroidery in the first task.

\section{Conclusion}

We may say that a positive shift in the attitude toward a mandatory subject may be accompanied by an improvement in understanding this subject. An attitude was defined by Hilgard \& Atkinson (1967) as "a favorable or unfavorable orientation toward an object, concept, or situation, as well as a readiness to react in a predetermined, consistent manner toward these objects, concepts, or situations or to those related to them." Attitudes develop through numerous learning experiences, and consequently individuals tend to favor activities that they believe they will or may be successful in. If individuals believe that they successfully completed previous, similar tasks, in all probability they will approach the next task with an emotionally positive approach. On the other hand, if they believe they have failed in previous, similar tasks, it is likely they will approach the next task in an unfavorable state of mind (Bloom, 1984).

Robert (1982) found a positive correlation between achievement in geometry and attitudes toward the subject: students with lower grades disliked the subject while students with higher grades viewed it favorably. Thus, it is possible to infer a bi-directional causal relationship, such that students like subjects that they have mastered and dislike learning subjects in which they lack proficiency. On the other hand, it is also possible to state that students master subjects that they like and do not master subjects that they dislike. In other words, there may be a circular effect, and this is where ethnomathematics enters as a solution to change students' attitudes to geometry. Ethnomathematics can facilitate a positive attitude of students toward geometry, as it makes the material more familiar and closer to home by integrating tools and elements that express mathematical val- 
ues from their daily lives in class. This will help students to grasp the complex rules and concepts of geometry, which will be reflected in their improved conception, comprehension, success and desire to study geometry.

Thus we may conclude by saying that in the instruction of geometry, educators should link the ethnomathematical approach with Van Hiele's theory, first by diagnosing the level of each student and then constructing an individual study plan for each student. This plan should provide and integrate cultural elements and tools from the students' daily lives at all levels of instruction, as an instructional strategy and means of illustration. If this is done, we will certainly improve the students' desire to study geometry as well as their achievements in this field.

\section{Conflicts of Interest}

The authors declare no conflicts of interest regarding the publication of this paper.

\section{References}

Aichele, D., \& Downing, C. (1985). Increasing the Participation of Native Americans in Higher Mathematics. Project funded by the National Science Foundation.

Amit, M., \& Abu Qouder, F. A. (2017). Weaving Culture and Mathematics in the Classroom: The Case of Bedouin Ethnomathematics. In M. Rosa et al. (Eds.), Ethnomathematics and Its Diverse Approaches for Mathematics Education (pp. 23-50). ICME-13 Monographs, Cham: Springer. https://doi.org/10.1007/978-3-319-59220-6_2

Amit, M., Fried, M. N., \& Abu-Naja, M. (2007). The Mathematics Club for Excellent Students as Common Ground for Bedouin and Other Israeli Youth. The Montana Mathematics Enthusiast, Monograph, 1, 75-90.

Bloom, B. S. (1984). The Two Sigma Problem: The Search for Methods as Effective as One-to-One Tutoring. Educational Researches, 13, 4-16.

https://doi.org/10.3102/0013189X013006004

Burger, W. F., \& Shaughnessy, J. M. (1986). Characterizing the van Hiele Levels of Development in Geometry. Journal for Research in Mathematics Education, 17, 31-48. https://doi.org/10.2307/749317

Chandler, P., \& Sweller, J. (1991). Cognitive Load Theory and the Format of Instruction. Cognition and Instruction, 8, 293-332. https://doi.org/10.1207/s1532690xci0804_2

Crowley, M. L. (1987). The Van Hiele Model of the Development of Geometric Thought. Teaching and Learning, K-12-1987 Yearbook. Virginia, USA: NCTM.

D’Ambrosio, U. (1984). Socio Cultural Basis of Mathematics Education-Plenary Address at the Fifth International Congress on Mathematical. Adelaide Australia: Education.

D’Ambrosio, U. (1987). Reflections on Ethnomathemathematics. International Study Group on Ethnomathemathematics. Newsletter, 3, 3-5.

D’Ambrosio, U. (2002). Ethnomathemathematics: An Overview. In M. de Monteiro (Ed.), Proceedings of Second International Conference on Ethnomathemathematic (pp. 3-5). (ICEM2), CD Room, Ouro Preto, Prazil: Lyrium Comunacacao Ltda.

Fuys, D., \& Geddes, D. (1984). An Investigation of Van Hiele Levels of Thinking in Geometry among Sixth and Ninth Grades. Research Findings and Implications. Paper presented at the Annual Meeting of the American Educational Research Association, New 
Orleans, 27 April 1984.

Gawlick, T. (2002). On Dynamic Geometry Software in the Regular Classroom. Zentralblatt für Didaktik der Mathematik, 34, 85-92. https://doi.org/10.1007/BF02655711

Gilmer, G. (1990). An Ethnomathematical Approach to Curriculum Development. International Study Group on Ethnomathematics. Newsletter, 5, 4-6.

Goldin, G. A., Epstein, Y. M., Schorr, R. Y., \& Warner, L. B. (2011). Beliefs and Engagement Structures: Behind the affective Dimension of Mathematical Learning. ZDM, 43, 547-560. https://doi.org/10.1007/s11858-011-0348-Z

Gutierrez, A., Jaime, A., \& Fortuny, J. M. (1991). An Alternative Paradigm to Evaluate the Acquisition of the van Hiele Levels. Journal for Research in Mathematics Education, 22, 237-251. https://doi.org/10.2307/749076

Hershkowitz, R., \& Vinner, S. (1983). On Concept Formation in Geometry. Zentralbatt feur Didactic der Mathematic, 1, 20-25.

Hilgard, E. R., \& Atkinson, R. C. (1967). Introduction to Psychology (4th ed.). New York: Harcourt.

Hoffer, A. (1981). Geometry Is More than Proof. The Mathematics Teacher, 74, 11-18. https://doi.org/10.5951/MT.74.1.0011 https://cms.education.gov.il/NR/rdonlyres/9066AD04-2DC3-4BE6-9D31-981391E7619 D/104162/TIMSS_2007_fullreport.pdf

Keitel, C., Damero, P., Bishop, A., \& Gerdes, P. (1989). Mathematics, Education, and Society. Science and Technology Education. Paris, France: UNESCO.

Keith, J. (2000). Teacher Knowledge and Professional Development in Geometry. Proceedings of the British Society for Research into Learning Mathematics, 20, 109-114. http://www.bsrlm.org.uk/IPs/ip20-3/BSRLM-IP-20-3-19.pdf

Lipka, J., Wong, M., \& Andrew-Ihrke, D. (2013). Alaska Native Indigenous Knowledge: Opportunities for Learning Mathematics. Mathematics Education Research Journal, 25, 129-150. https://doi.org/10.1007/s13394-012-0061-4

Mayberry, J. W. (1983). The Van Hiele Levels of Geometric Thought in Undergraduate Preservice Teachers. Journal for Research in Mathematics Education, 14, 58-69. https://doi.org/10.2307/748797

NCTM (2000). Principles and Standards for School Mathematics. NCTM.

Pollock, E., Chandler, P., \& Sweller, J. (2002). Assimilating Complex Information. Learning and Instruction, 12, 61-86. https://doi.org/10.1016/S0959-4752(01)00016-0

Powell, A. B., \& Frankenstein, M. (Eds.) (1997). Ethnomathematics: Challenging Eurocentrism in Mathematics Education. Albany, NY: State University of New York Press.

Robert, P. L. (1982). The Relationship of Attitude, Achievement and IntroversionExtraversion among High School Student in Euclidean and Transformational Geometry Classes. Ph.D. Thesis, New York: Fordham University.

Senk, S. L. (1985). How Well Do Students Write Geometry Proofs? The Mathematics Teacher, 78, 448-456. https://doi.org/10.5951/MT.78.6.0448

Sullivan, S., \& Glanz, J. (2004). Supervision That Improves Teaching: Strategies and Techniques (2nd ed.). Thousand Oaks, CA: Corwin Press.

Swafford, J. O., Jones, G. A., \& Thornton, C. A. (1997). Increased Knowledge in Geometry and Instructional Practice. Journal for Research in Mathematics Education, 28, 467-483. https://doi.org/10.2307/749683

Sweller, J. (1988). Cognitive Load during Problem Solving: Effects on Learning. Cognitive Science, 12, 275-285. https://doi.org/10.1207/s15516709cog1202_4 
Sweller, J., van Merrienboer, J. J. G., \& Paas, F. G. W. C. (1998). Cognitive Architecture and Instructional Design. Educational Psychology Review, 10, 251-296.

https://doi.org/10.1023/A:1022193728205

Thomas, J. W. (2000). A Review of Research on Project-Based Learning. San Rafael, CA: Autodesk Foundation.

TIMSS (2007). http://www.bsrlm.org.uk/IPs/ip20-3/BSRLM-IP-20-3-19.pdf

Usiskin, Z. (1982). Van Hiele Levels and Achievement in Secondary School Geometry (Final Report). Chicago, IL: Department of Education, University of Chicago.

Van Hiele, P. M. (1959). Development and the Learning Process. Acta Pedagogical Ultrajectra (pp. 1-31). Groningen: J. B. Wolters.

Van Hiele, P. M. (1987). Van-Hiele Levels, a Method to Facilitate the Finding of Levels of Thinking in Geometry by Using the Levels in Arithmetic. In M. M. Lindquist (Ed.), The National Council of Teachers of Mathematics (pp. 1-16). Reston, VA: National.

Verner, I., Massarwe, K., \& Beshouty, D. (2013). Constructs of Engagement Emerging in an Ethnomathematically-Based Teacher Education Course. Journal of Mathematical Behavior, 32, 494-450. https://doi.org/10.1016/j.jmathb.2013.06.002

Vinner, S. (1976). The Naive Concept of Definition in Mathematics. Educational Studies in Mathematics, 7, 413-429. https://doi.org/10.1007/BF00452223

Vinner, S. (1977). The Concept of Exponentaion at the Undergreduate Level and the Definition Approach. Educational Studies in Mathematics, 8, 17-26.

https://doi.org/10.1007/BF00302501 